\title{
Development of Adhesion Force Evaluation Equipment for Nano Diamond Coated Tool Using Shear Method
}

\section{Jinghua Li ( 100080@naver.com)}

Kumoh National Institute of Technology https://orcid.org/0000-0002-2725-2086

\section{SoJin Lee}

Kumoh National Institute of Technology

HyunKyu Kweon

Kumoh National Institute of Technology

\section{Original Article}

Keywords: Nano-diamond Coated Tool, Thin-film, Adhesion Force, Frictional Force, Characteristic,

Vibration

Posted Date: April 21st, 2020

DOl: https://doi.org/10.21203/rs.3.rs-22825/v1

License: (a) (1) This work is licensed under a Creative Commons Attribution 4.0 International License. Read Full License

Version of Record: A version of this preprint was published at Measurement and Control on December 4th, 2020. See the published version at https://doi.org/10.1177/0020294020977890. 


\title{
DEVELOPMENT OF ADHESION FORCE EVALUATION EQUIPMENT FOR NANO DIAMOND COATED TOOL USING SHEAR METHOD
}

\author{
JingHua Li ${ }^{1}$, SoJin Lee ${ }^{1}$ and HyunKyu Kweon ${ }^{1}$ \\ ${ }^{1}$ Department of Mechanical System,Kumoh National Institute of Technology Gumi, Republic of Korea, hkkweon@kumoh.ac.kr
}

\begin{abstract}
In this study, we have developed a thin film adhesion evaluation system for diamond coating tool, which is mainly used for CFRP processing, which is widely used in aviation and automobile industries. Carbon fiber reinforced plastic (CFRP), which is a composite material is widely used for high efficiency and light weight in the aerospace industry and automobile industry. CFRP has a low specific gravity and a high specific strength. However, it has difficulty in processing because it has difficulty property. In order to cope with this problem, CFRP is processed using a diamond coating tool with a higher surface hardness. Because of the high surface hardness of the diamond coating tool, it is difficult to evaluate the adhesive strength of the tool, and it is difficult to quantitatively evaluate the existing adhesion force evaluation method and it is costly. This study has developed an evaluation method to measure the adhesive force based on the data of the grinding process using a low cost polishing pad. When the frictional force and the shearing force are applied to the specimen by the sanding belt, friction is continuously generated between the coating layer and the belt, and peeling occurs at the moment when a specific load is applied. Acceleration, load, and torque values that occur during each experiment are collected through acceleration sensors, load cells, and torque sensors. The data obtained through the experiments are subjected to FFT processing and analysis. As a result, the peeling point and the critical load value at this point are identified and referred to as the adhesion force of the coating layer.
\end{abstract}

Keywords: Nano-diamond Coated Tool, Thin-film, Adhesion Force, Frictional Force, Characteristic, Vibration

\section{Introduction}

Among the carbon fiber composite materials, the carbon fiber reinforced plastic (CFRP), which is a plastic material, is lightweight, has excellent strength and rigidity and has been used in the aerospace and defense industries. In recent years, efforts have been made to improve fuel efficiency by using lightweight materials to reduce carbon dioxide emissions and solve global warming problems, especially in transport equipment. Accordingly, there is a growing demand for carbon fiber-reinforced plastics, which are necessary mate- 
rials for lighter weight. However, the characteristics of CFRP vary depending on the properties of carbon fiber, the direction of lamination, the weaving method, and the anisotropy in which the mechanical properties vary greatly depending on the direction.

In order to solve the problem of CFRP, which is a typical roughing material, cutting of fine cutting fiber, cutting of a fine cutting fiber into a rotating tool, peeling of a fibrous layer is used [1-3]. Diamond-coated tools are coated in the manner of Micro Crystalline Diamond (MCD) and Nano Crystalline Diamond (NCD). In this paper, NCD drill was selected as the specimen.

An important factor in determining the performance and durability of diamond coated tools is the adhesion between the base material and the coating layer. The adhesive force is defined as the critical load at the time of peeling, and there are various methods such as scratch test, indentation test, and tape test. The most widely used test method is the scratch test. In the scratch test, a force is applied to diamond particles to leave scratch marks on the coating layer, and the adhesion force is measured by analyzing the scratch marks. However, there are many factors that determine the adhesion of the coating layer such as the moving speed of the indenter, the thickness of the coating layer, and the biggest problem is that quantitative values are not obtained[4] and the expensive cost of the diamond indenter is high.

In this paper, we developed a new adhesion evaluation method to measure the adhesion between the coating layer and the base metal, quantify the adhesion of the coating layer, and reduce the test cost by using a polishing pad as a substitute for expensive diamond indenter with short life span.

\section{Ease of Use (Heading 2)}

Fig. 1 shows the equipment developed by applying the friction and wear tester. The experimental equipment consists of a load applying part, a specimen fixing and moving part, a sanding belt driving part and a measuring part.

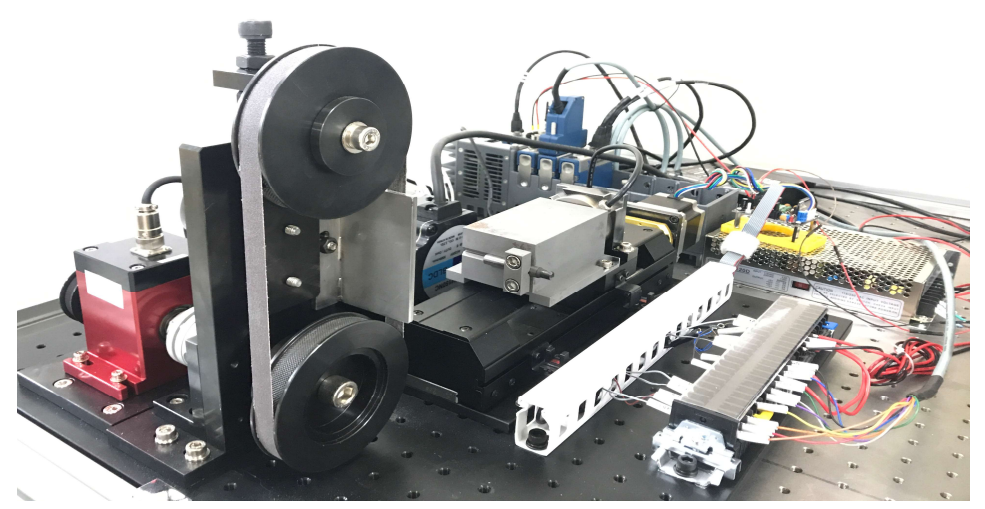

Figure 1. Shear condition Equipment.

The purpose of the development of this equipment is to remove the diamond coating layer of the specimen NCD tool from the base material and obtain the critical load at that 
time.

The load acting in the direction perpendicular to the line in contact with the NCD tool and the sanding belt during the test is defined as the vertical load. This vertical load increases linearly up to a certain upper limit during the experiment and then decreases. As the load is applied to the specimen, the load and frictional force acting on the specimen increase and the coating layer peels off.

\section{Experimental Results and Analysis}

\subsection{Adhesive force measurement experiment}

In this experiment, the coating layer of the specimen is peeled off by using the sanding belt while increasing the force applied to one point of the NCD tool specimen. The purpose of this study is to confirm the time of peeling and the critical load and frictional force at the point of peeling of the coating layer during the experiment.

\subsection{Sample test}

Fig. 2 shows the load, torque, and friction data generated during the test of the flute part. Fig. 3 is the coefficient of friction obtained by using load data and friction force. It can be seen that the coefficient of friction decreases from 0.6 to 0.3 , unlike the experimental results of the shank portion where the coefficient of friction varies from 0.3 .

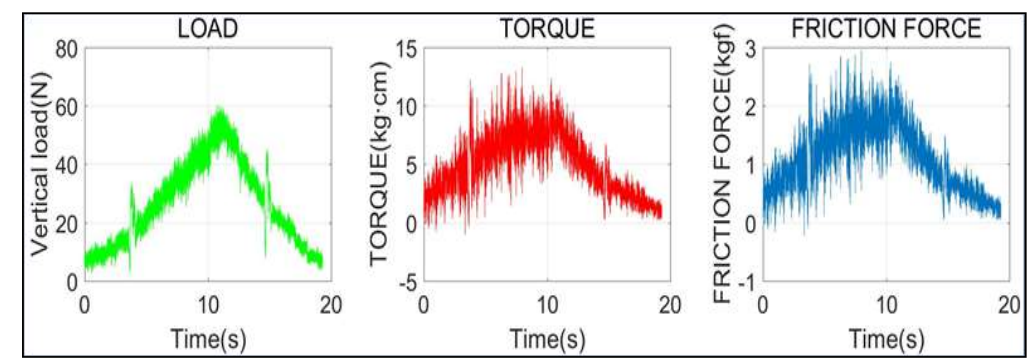

Figure 2. Flute part experimental result. 


\subsection{Data processing}

Experimental program was developed based on LabVIEW program of National Instruments to be able to experiment under various experimental conditions using experimental equipment. It includes BLDC motor and step motor speed adjustment, load upper limit setting and automatic test function. It can monitor acceleration, load and torque value in real time.

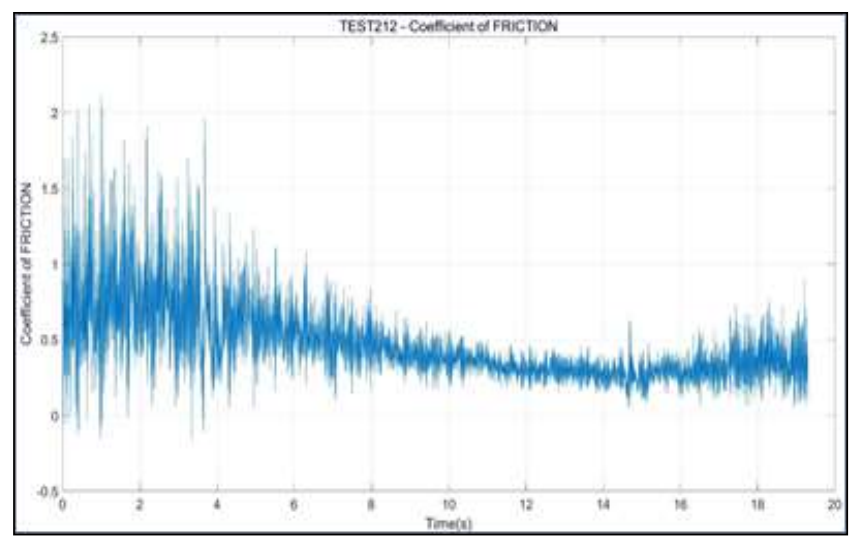

Figure 3. Flute friction coefficient.

We have developed a data processing program based on MATLAB to detect the peeling point by processing and analyzing the data obtained through experiments. The processing of load, torque and acceleration data obtained through experiment. The friction force and the friction coefficient can be obtained by using the load data and the torque data passed through the filter. The acceleration data obtained by attaching the single-axis acceleration sensor to the Z-axis where the vibration is greatest is cut at intervals of 1 second every 0.1 second to cover the Hamming window. This data is subjected to FFT analysis to detect the peeling point at which the coating layer is peeled off, obtain the load at the peeling point from the load graph, and obtain the frictional force from the frictional force graph. This critical load and frictional force are called adhesion forces.

\subsection{Peeling moment}


First, as shown in Fig. 4, the front part is divided into 'A-load increasing section' and the rear part is called 'B-load reducing section' based on the maximum value of the load applied to the specimen. When the tool surface was checked after the test, if peeling occurred, it could be deduced that peeling occurred in area A where the load increased. Therefore, in the region A, the NCD coating layer is vibrated due to friction, and there is a section where the vibration characteristic is changed into vibration due to wear of the tungsten carbide due to exposure of the base material after peeling. In the region $\mathrm{B}$, peeling has already occurred in the region A, so that only the vibration characteristic due to wear of the tungsten carbide occurs.

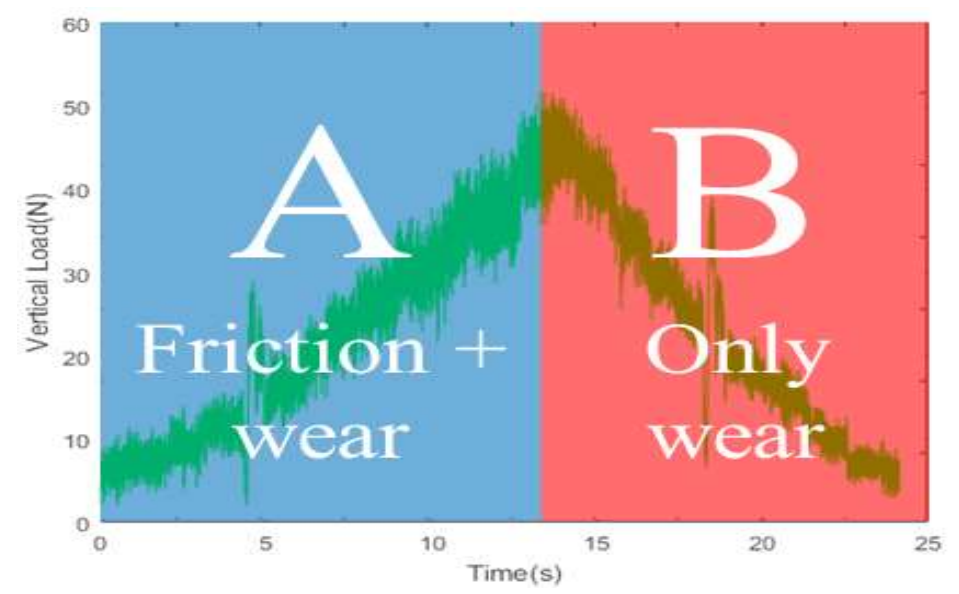

Figure 4. Classification of load change area.
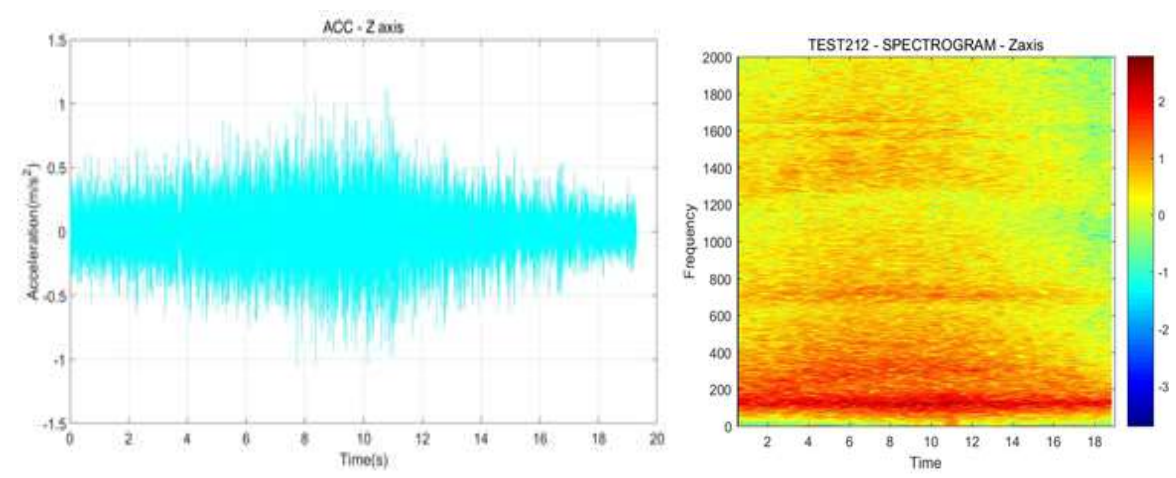

Figure 5. Flute acceleration data and Flute Spectrogram.

Fig. 5 shows the Z-axis acceleration data generated during the Flute part experiment and the spectrogram after Fourier transform. It can be seen that the strength in the vicinity is strongly shown throughout the experiment. Unlike the experimental results of the shank section, it can be seen that the frequency intensities of the sections A and B are not symmetrical throughout the experiment. This means that the characteristics of the specimen surface changed from the NCD coating layer to the base material during the experiment. 
From the spectrogram data of the Shank part and the Flute part experiment, it can be obtained as shown in Fig. 6. This is a graph in which the average value of the frequency intensities in section A and section B is averaged.

Since the shank part has only the abrasive wear characteristics of tungsten carbide in both the A section and the B section, it can be confirmed that the frequency characteristics in the A section and the B section are almost the same.

However, it can be seen that the frequency characteristics of the A and B sections are different because the friction of the NCD coating layer and abrasion of the base material occur in the section A and only wear of the base material occurs in the section B.

It can be judged that the peeling occurs at the point where the characteristic of the frequency changes from the friction of the coating layer to the abrasion of the base material.

In order to detect the peeling point at which the characteristics of frequency are changed, the relative error is obtained from the FFT average data of the section B and the FFT data of the sections A and B having the frequency characteristics of the wear in the shank sec-
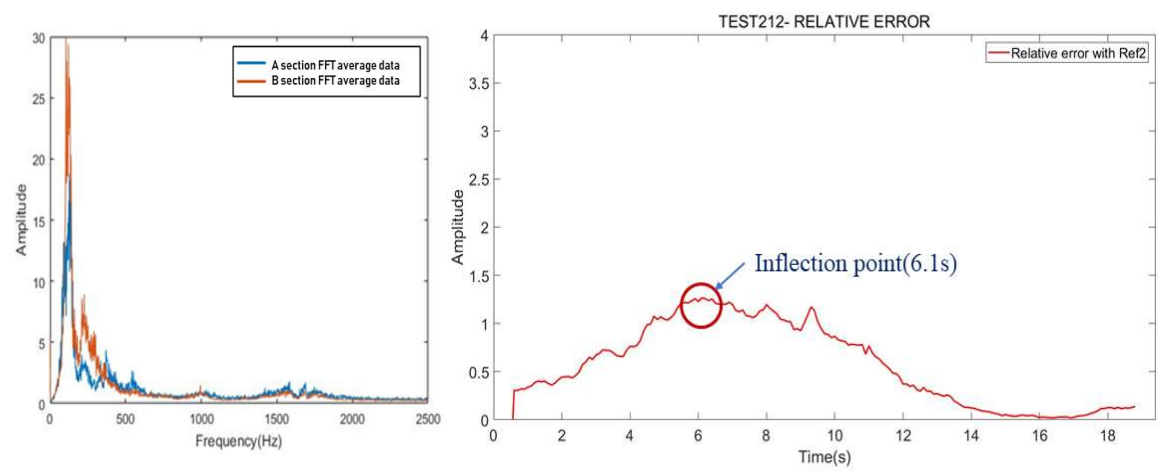

Figure 6. Flute Part A and B section FFT average data and Flute Relative error graph.

tion test results.

The relative error graph is shown in Fig. 6. The relative error increases gradually and then decreases again with the maximum value around 6.1 seconds. At this inflexion point, the frequency characteristic is changed from abrasion and adhesion wear of the coating layer to grinding wear of the base material.

The critical load and the frictional force at the time of peeling can be found from the following load graph and the frictional force graph. The critical load and the frictional force is $26.5 \mathrm{~N}$ and $15.92 \mathrm{~N}$.

\section{Conclusion}

In this study, we have developed a new adhesion force evaluation system by applying friction and wear tester to measure the adhesion of NCD tool coating layer and proposed a method to quantitatively measure the adhesion force by processing collected data from adhesion test. 


\section{References}

[1] S.J. Won, T.J. Ko, K.M. Park, I.C. Yoon, "Occurrence of Burrs At The Hole Exit Region of CFRP Drilling”, Proceedings of the KSMPE Conference, p.48, 2015.

[2] M.G. Gang, G.H. Kim, K.W. Shin, A.M. Jeong, H.Y. Kim, C.H. Kim, S.W. Lee, T.G. Kim, "Mechanical Cutting Process Trends for Difficult-to-Cut Materials", Journal of the Korean Society for Precision Engineering, Vol.35, No.3, pp.253-267, 2018.

[3] H.S. Kim, S.B. Kim, T.Y. Kim, S.H. Oh, "A Study on the Cutting Property of the Drill according to the Stacking Sequence of the CFRP", Proceedings of the KSMPE Conference, pp.154-158, 2011.

[4] G.H. Lee, "Adhesion measurement of thin films", The Korean Institute of Surface Engineering, p.9, 1989 .

\section{Availability of data and materials}

The data during the current study are available from the corresponding author on reasonable request.

\section{Competing interests}

The authors declare that there are no conflicts of interest regarding the publication of this paper.

\section{Funding}

Not applicable

\section{Authors' contributions}

$\mathrm{JH}$ and SJ were in charge of all experiments and wrote the manuscript; HK was responsible for the research idea of the Blast condition method and provided necessary help with all parts of this article. All authors read and approved the final manuscript.

\section{Acknowledgements}

This study was supported by the research fund of the Ministry of Trade, Industry \& Energy Industrial Technology Innovation Project. (Project Number: 10067069) 
Figures

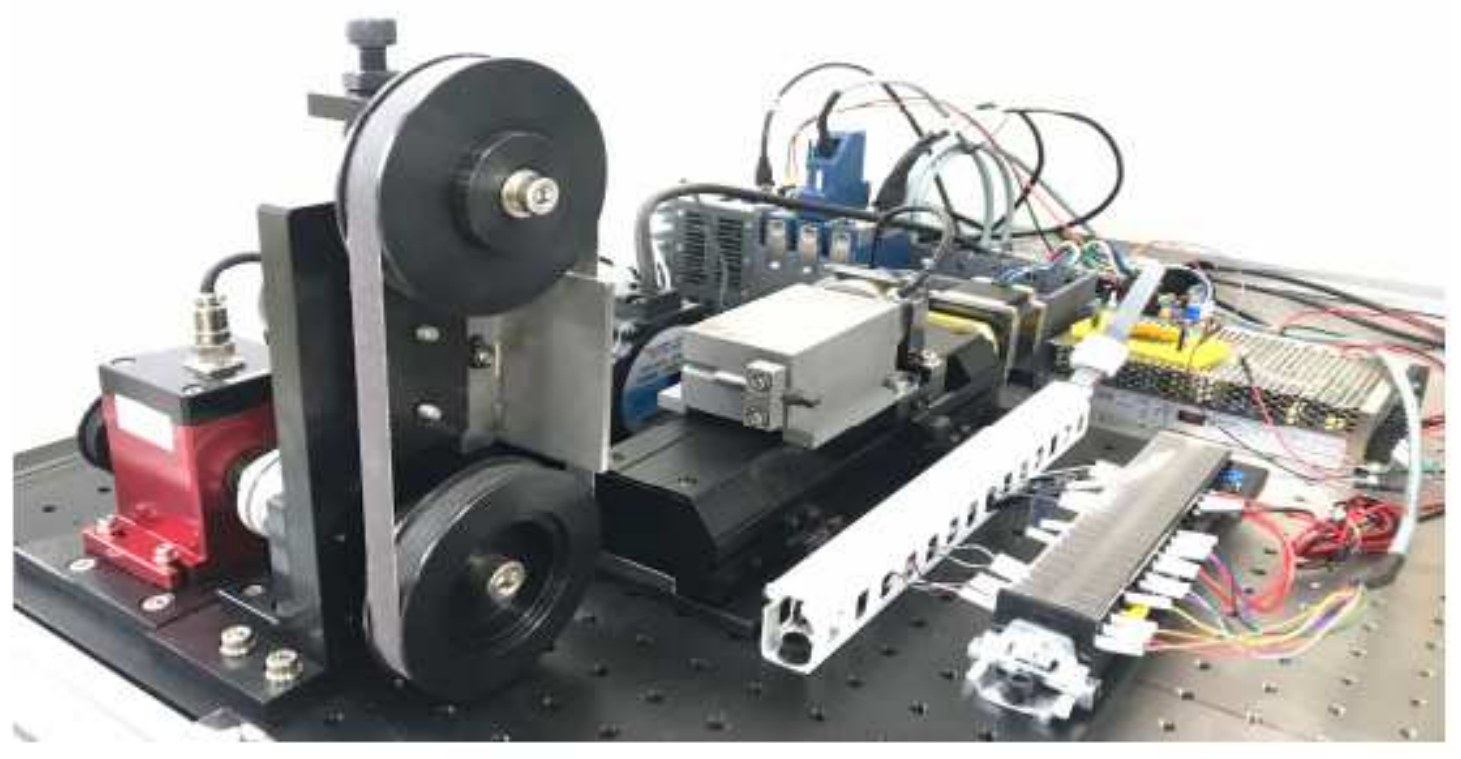

Figure 1

Shear condition Equipment.

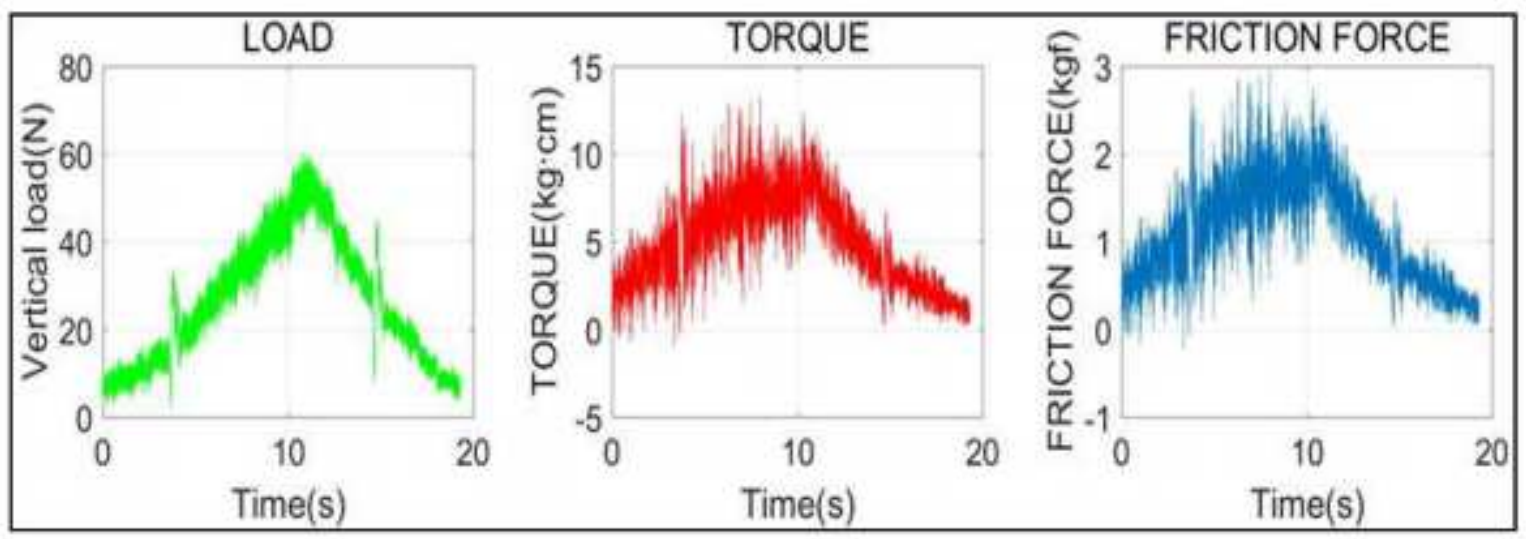

Figure 2

Flute part experimental result. 


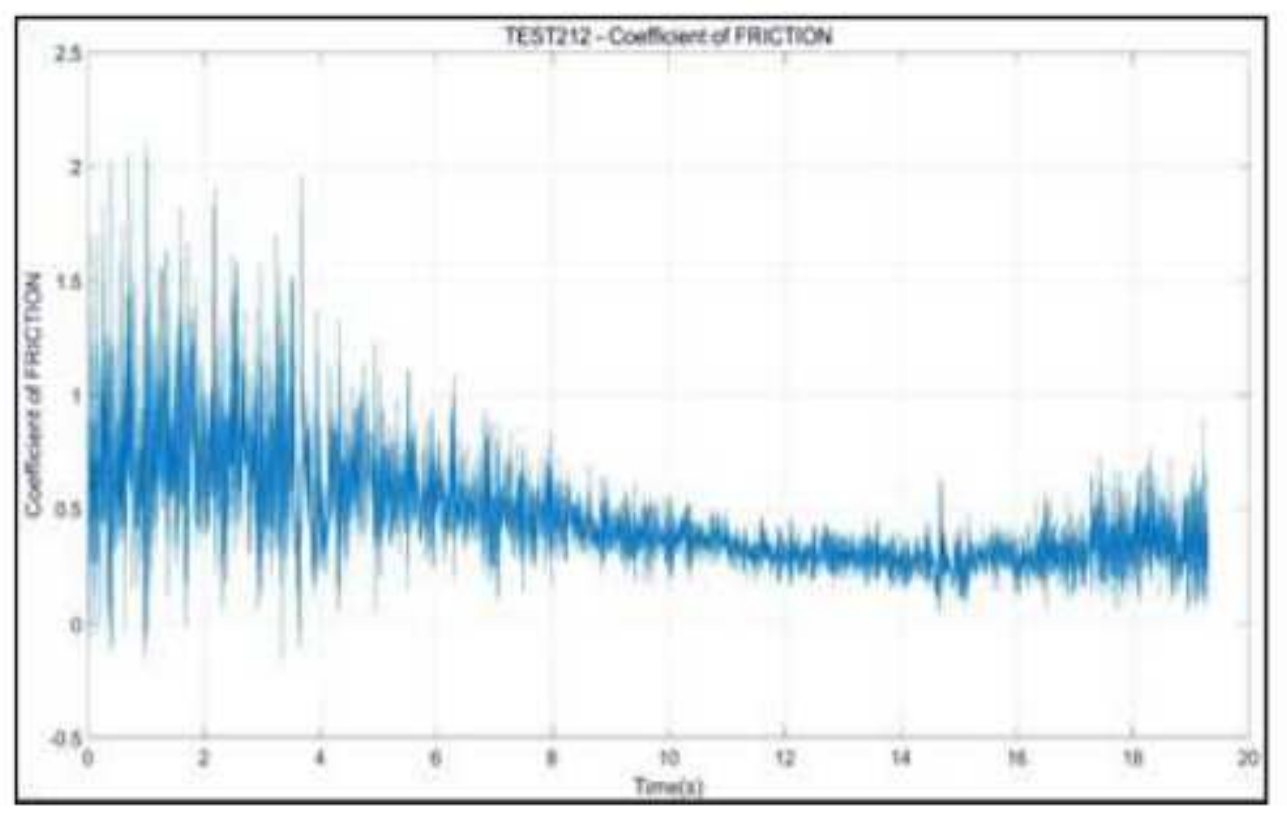

Figure 3

Flute friction coefficient.

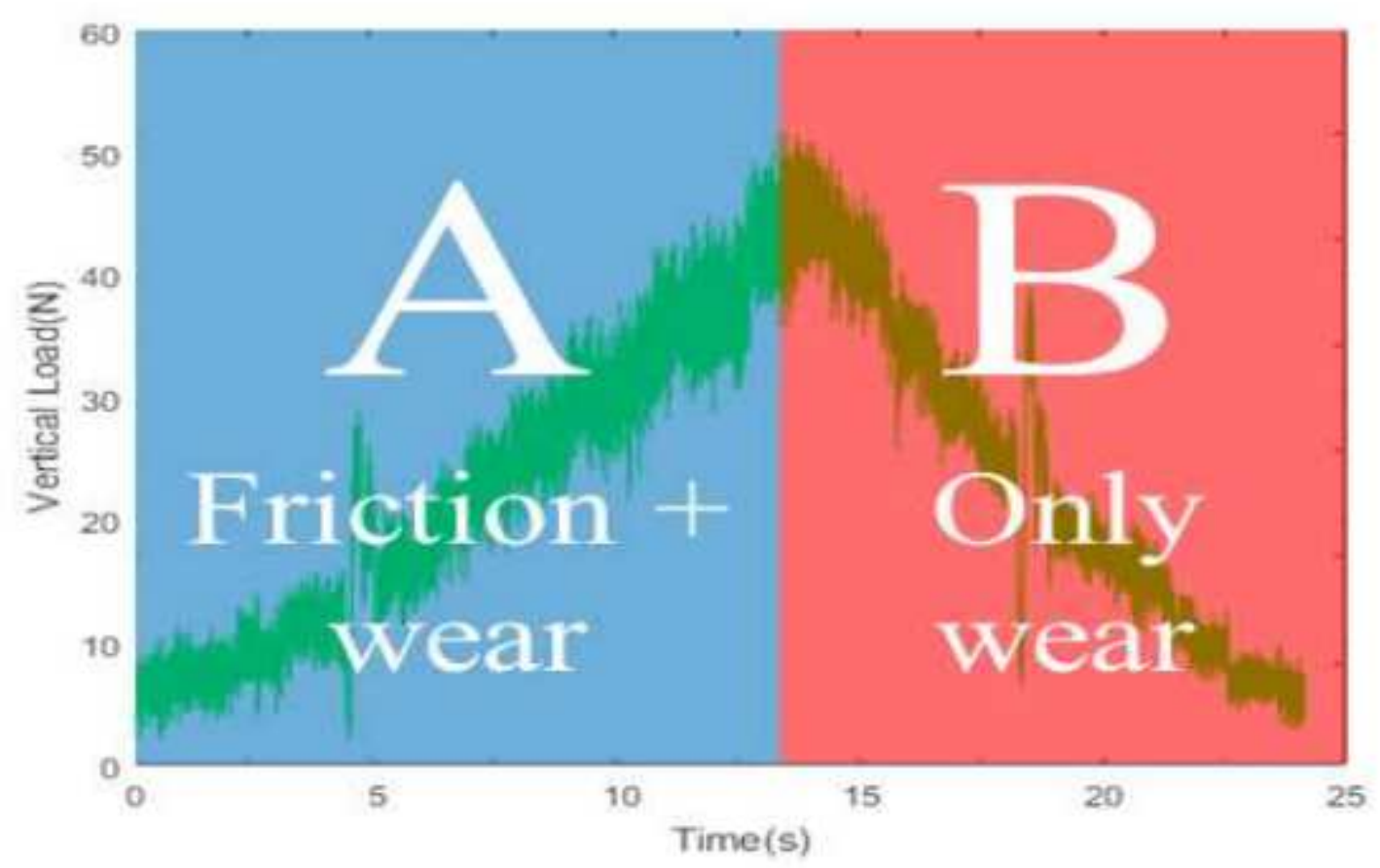

Figure 4

Classification of load change area. 

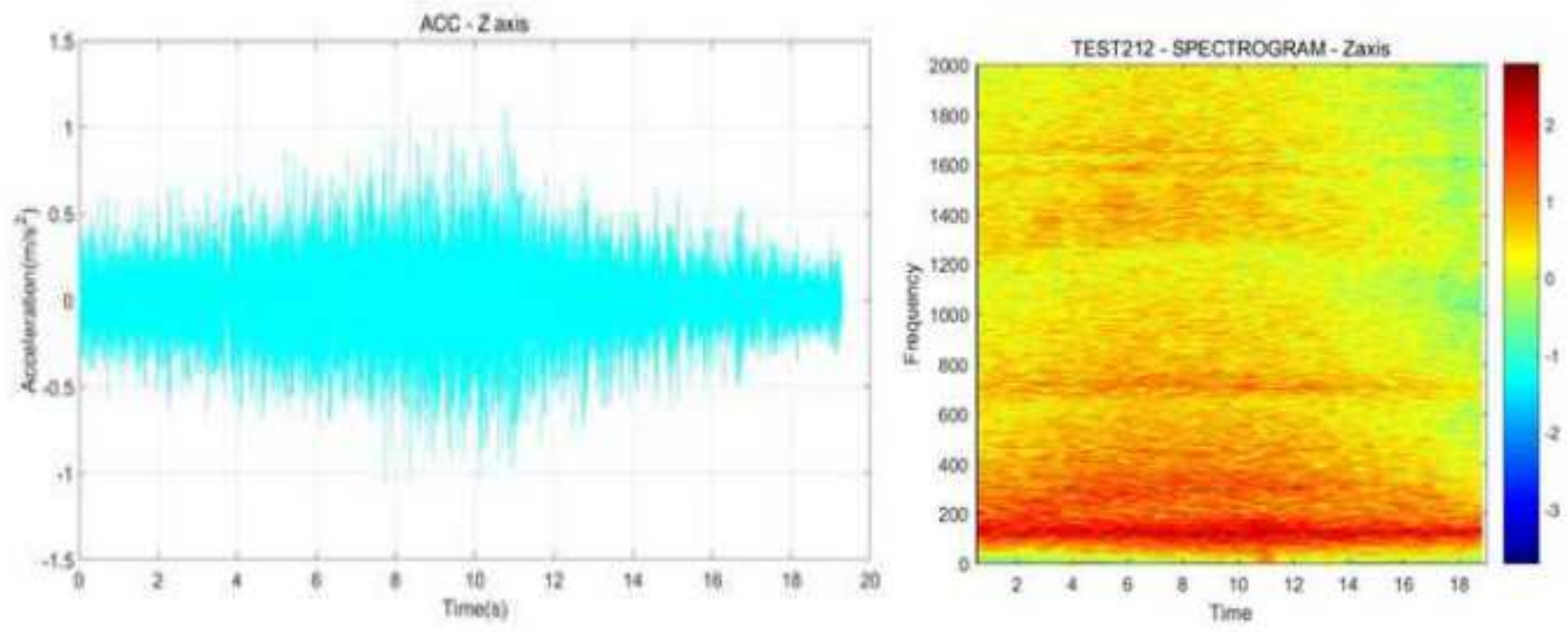

\section{Figure 5}

Flute acceleration data and Flute Spectrogram.
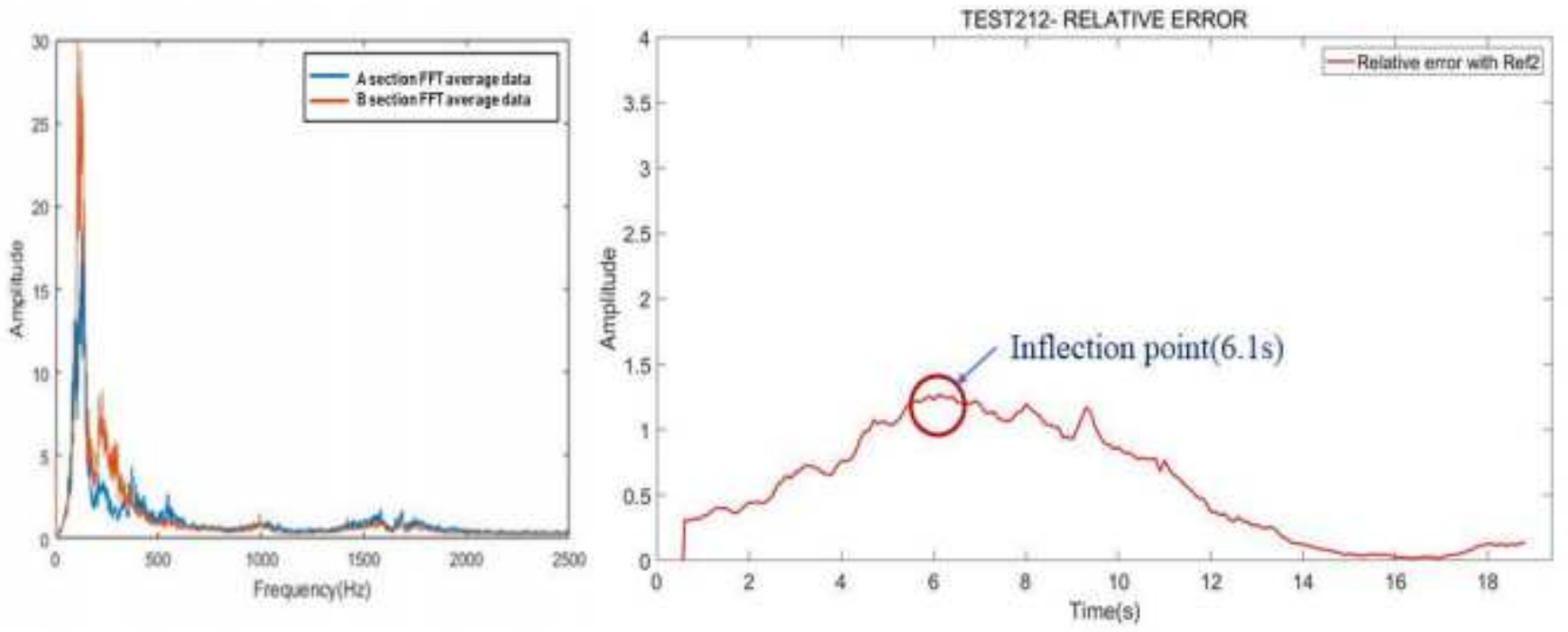

Figure 6

Flute Part A and B section FFT average data and Flute Relative error graph. 